\title{
Refractive index and salinity sensors by gallium-doped zinc oxide thin film coated on side-polished fibers
}

\author{
Chuen-Lin TIEN $^{1,2 *}$, HaO-Sheng MAO ${ }^{1}$, Tzu-Chi MAO $^{2}$ \\ ${ }^{1}$ Department of Electrical Engineering, Feng Chia University, Taichung, Taiwan \\ ${ }^{2}$ Ph.D. Program of Electrical and Communications Engineering, \\ Feng Chia University, Taichung, Taiwan
}

*Corresponding author: cltien@fcu.edu.tw

\begin{abstract}
This work presents a high-sensitivity refractive index and salinity sensor by using fiber-optic side -polishing and electron-beam evaporation techniques. Thin film coated on the flat surface of side -polished fibers can generate a lossy mode resonance (LMR) effect. A gallium-doped zinc oxide (GZO) thin film was prepared by an electron-beam evaporation with the ion assisted deposition method. The residual thickness of the side-polished fiber was $76.5 \mu \mathrm{m}$, and GZO film thickness of $69 \mathrm{~nm}$ was deposited on the flat surface of the side-polished fiber to fabricate LMR-based fiber sensors. The variation in the optical spectrum of LMR-based fiber sensors was measured by different refractive index saline solutions. The LMR wavelength shift is caused by the refractive index change, which is nearly proportional to the salinity. The corresponding sensitivity of the proposed fiber-optic sensor was $3059 \mathrm{~nm} / \mathrm{RIU}$ (refractive index unit) for the refractive index range of 1.333 to 1.398 . To evaluate the sensitivity of LMR salinity sensors, the saline solution salinities of $3.6 \%$, $7.3 \%, 10.9 \%, 14.6 \%, 18.2 \%$ and $21.9 \%$ were measured in this work. The experimental result shows that the sensitivity of the proposed salinity sensor is $9.94 \mathrm{~nm} / \%$.
\end{abstract}

Keywords: refractive index, thin film, side-polished fiber, lossy mode resonance, gallium-doped zinc oxide.

\section{Introduction}

Miniaturization of optical sensors is a key issue for effectively using them in fiber-optic sensing applications. The refractive index (RI) measurement has been widely applied in many fields, such as used in biological, chemical, and environmental engineering fields. Optical fiber sensors have the advantages of small size, low transmission loss, low cost, high sensitivity, portability and anti-electromagnetic interference [1]. Over the past few years, different liquid refractive index measurements have been reported in the literature. For example, TAHHAN et al. [2] reported the etched D-shaped FBG which 
was then coated with $\mathrm{TiO}_{2}$ nanostructured layer to sense deionized water and saline. The detected sensitivity was $1.257 \mathrm{~nm} /$ RIU for air-deionized water and $0.857 \mathrm{~nm} / \mathrm{RIU}$ for air-saline. In 2014, Li et al. [3] presented a polyelectrolyte multilayer (PEM) coated on the long-period fiber grating (LPFG) used as RI sensor with sensitivity of about $789.47 \mathrm{~nm} / \mathrm{RIU}$ which is only sensitive to an extremely small range of 1.400-1.453. In 2017, HASAN et al. [4] presented a highly sensitive photonic crystal fiber (PCF) refractive index sensor based on the surface plasmon resonance (SPR) effect. They used numerical investigations to show the wavelength sensitivity of $10800 \mathrm{~nm} / \mathrm{RIU}$ and amplitude sensitivity of $514 \mathrm{RIU}^{-1}$ in the sensing range between 1.46 and 1.48. In the same year, UsHA and GUPTA [5] presented the lossy mode resonance (LMR) of zinc oxide $\mathrm{ZnO})$-coated optical fiber as a refractive index sensor. Their experimental results show nanostructured LMR sensors enhanced sensitivity in comparison to a bulk layered probe. However, the previous techniques exhibit a smaller detection range for RI changes.

Simply speaking, the salinity is the quantity of dissolved salt content of the water. The salinity is an important parameter to evaluate the density of seawater. The salinity is expressed in grams per kilogram of seawater, that is, the salinity unit is in parts per thousand (\%) [6]. Over the past few years, a great deal of indirect methods have been reported for salinity measurement. In 1994, JIN et al. [7] proposed an intensity-modulated fiber-optic sensor based on radiation loss for the salinity measurement. In 1999, DinIz et al. [ [ $]$ reported a polyaniline matrix coated wire electrode for salinity measurement in a range from $0.010 \%$ to $75 \%$. In 2002 , ZHAO and LIAO [9] reported an optic fiber sensor developed for simultaneous seawater temperature and salinity measurement. The sensor exploits beam deviation caused by refraction due to the salinity of seawater at the receiving end face of the optical fiber array. They also reported an optical fiber sensor for remote monitoring of salinity in water [10]. The salinity is measured by a position-sensitive detector. In 2008, MeN et al. [11] proposed a fiber-optic sensing system consisting of FBGs coated with different polymers responding to variations of both temperature and salinity. The temperature and the salinity sensitivities of the polyimide-coated grating were $0.0094 \mathrm{~nm} /{ }^{\circ} \mathrm{C}$ and $0.0165 \mathrm{~nm} / \mathrm{M}$, respectively. In 2018 , QIAN et al. [12] reported a review of the salinity measurement techniques based on the optical fiber sensor. They compared performances of various sensing structures and analyzed the advantages and disadvantages of different sensors. In order to increase the sensitivity and scale down the size of sensors, GZO thin film coated on a side-polished fiber has been proposed in this work.

Optical fiber sensors have been used extensively in different engineering fields due to their many desirable advantages [13]. Compared to traditional sensors, these have better sensitivity, accuracy, and reliability. The fiber-based sensors possess the potential of high sensitivity, such as the side-polished fiber sensor [14], thin-film type fiber sensor [15], Bragg grating fiber sensor [16] as well as the long-period grating fiber sensor [17]. Among them, a side-polished fiber is fabricated by removing the cladding of the optical fiber to expose the evanescent field to the external medium. The interaction between the evanescent wave and the external medium will result in an optical transmission loss of the side-polished fibers. Therefore, we used the side-polished 
fibers to increase the evanescent field magnitude and sensitivity. In addition, thin film coated on the side-polished fibers can be used as a resonance-based fiber sensor. The phenomenon of thin-film coatings combined with optical fiber devices can produce two different resonance types: surface plasmon resonance (SPR) [18] and lossy mode resonance (LMR) [19], also named guided mode resonance or $\mathrm{TE}_{0}$ and $\mathrm{TM}_{0}$ resonances [20]. The well-known resonance phenomenon caused by these modes is surface plasmon resonance (SPR). The SPR sensor was based on the utilization of the Kretschmann-Raether configuration [21], which consists of an optical prism on which a metallic thin film coating is deposited. However, there are other types of modes supported by absorbing thin films. YANG and SAMBLES [22] reported that a thin film supports a lossy mode resonance (LMR) effect if the real part of its permittivity is positive and higher in magnitude than both its own imaginary parts and the material surrounding the thin film. The evanescent field of a propagation light wave in a side-polished fiber is accessible through a removed section of the cladding. LMR is considered as the standing electromagnetic wave confined between the two surfaces of the waveguide. For example, transparent conductive oxides (TCO) are good candidates for supporting LMR thanks to the combination of conductive and transparent properties in the visible/ infrared region. Gallium-doped $\mathrm{ZnO}$ (GZO) films, with low resistivity and high transmittance in the visible and near infrared spectrums, have been prepared by different techniques [23-26]. Among the various techniques, electron-beam evaporation with ion beam deposition (IAD) offers dense, uniform, and well-adhered films. In this work, we chose GZO thin film as a sensing material coated on the side-polished fibers that produces the LMR phenomenon.

Due to the liquid refractive index changes being proportional to its density, which is strongly correlated with salinity, the measurement of seawater's refractive index can be used to measure its salinity. Little research has been done on the measurement of salinity concentration and the refractive index of saline solutions. Therefore, we proposed a LMR fiber-optic sensor that combines the side-polishing fiber and GZO thin film coatings. GZO thin films, with different thicknesses, were deposited on the side-polished fibers. In this work, the influence of the film thickness on the sensitivity of a side-polished fiber sensor was also presented.

\section{Method}

The partly plastic cladding of SMF was removed by a homemade polishing machine to form side-polished fibers, and then ultrasonically washed in detergent, deionized water, piranha solution and finally deionized water. The side-polished fibers were placed into a dual electron-beam evaporation chamber and coated with a single layer GZO thin film. GZO thin film was prepared by the electron-beam evaporation technique and ion beam deposition method. One advantage of this process is the fact that it permits direct transfer of energy to a source during heating and that it is very efficient in depositing pure evaporated material to substrate. An additional benefit of e-beam evaporation is higher deposition rates than possible with either sputtering or resistive 
evaporation. When a side-polished fiber is coated with a thin-film material, it can be regarded as a wave-guide structure, and the propagation of light is affected. The surface plasmon resonance (SPR) phenomenon occurs when the real part of the thin-film permittivity is negative and higher in magnitude than both its own imaginary parts and the permittivity of the material surrounding the thin film. In this case, coupling occurs between light propagating through the wave-guide and a surface plasmon. It is well known that the SPR can only be generated by a TM polarized light source. On the other hand, a lossy mode resonance (LMR) effect occurs when the real part of the thin-film permittivity is positive and higher in magnitude than both its own imaginary parts and the permittivity of the material surrounding the thin film. Unlike the SPRs, LMRs can be generated with either TE polarized or TM polarized light [27]. Moreover, multiple resonances can be obtained without modifying the optical fiber geometry, and they can be obtained with a wide variety of materials, such as metal oxide, polymer, and semiconductors. Some studies demonstrated that the propagation of light in semiconductor cladding waveguides exhibits some attenuation maxima for specific thickness values of the semiconductor cladding and at certain wavelengths of incidence values [28]. This is due to a coupling between the waveguide modes and a specific lossy mode of the semiconductor layer [29].

When thin films are coated onto side-polished fibers, the propagation of light is changed. Different types of electromagnetic resonances can be observed depending on the dielectric properties of thin films. Less studies of electromagnetic resonance are devoted to LMR effect. In 2010, LMR-based fiber sensors were first demonstrated experimentally and applied in refractive index sensing [미]. LMRs have been observed with metal oxides, such as indium tin oxide (ITO) [31], $\mathrm{TiO}_{2}$ [32, 33], and indium oxide [34]. The Drude-Lorentz model [35] can be used to represent the dielectric constant of GZO thin films and the permittivity of a lossy material is given by

$$
\varepsilon(\omega)=\varepsilon_{\infty}-\frac{\omega_{\mathrm{p}}^{2}}{\omega^{2}+i \omega / \tau}+\frac{s_{\mathrm{o}} \omega_{\mathrm{o}}^{2}}{\omega_{\mathrm{o}}^{2}-\omega^{2}-i \gamma \omega}
$$

where $\varepsilon_{\infty}$ is the high frequency dielectric constant, and the literature value of $\varepsilon_{\infty}$ is about $3.5-3.7[\underline{36}] ; \omega$ is an angular frequency of a time-dependent electric field and $\omega_{\mathrm{p}}$ is the plasma frequency; $\tau$ is the electronic scattering time; $s_{\mathrm{o}}$ is the oscillator strength, $\omega_{\mathrm{o}}$ is the oscillator resonance frequency, and $\gamma$ is the oscillator damping constant.

The complex dielectric constant, it is given by a real part and an imaginary part. The relative permittivity can be expressed in terms of a complex refractive index $N$ as

$$
N=\sqrt{\varepsilon}=n+i k
$$

where $n$ and $k$ are the real and imaginary parts of the complex refractive index of the thin film material, respectively. The interaction of light at the cladding-film interface will result in the rearrangement of the guided modes propagating through the GZO-coated side-polished fiber. In the theoretical aspects, if the phase matching condition is satisfied, then the propagation constant of a guided mode and that of lossy mode are equal. 
If GZO thin film is coated on the flat surface of a side-polished fiber (as a coating substrate), the real part of the complex refractive index is positive and higher in magnitude than its own imaginary part and that of the surrounding medium, then the LMR can be observed in this structure. Thus GZO thin film coated onto side-polished fibers to form a sensing structure will support dielectric waveguide modes. However, these modes will be lossy because of the imaginary part of the complex refractive index of GZO films. Furthermore, the dispersion curves for the proposed sensing structure (i.e. side-polished fiber/GZO thin film/surrounding medium) will be similar to dielectric waveguides. Consequently, there is a coupling between the guide mode and the lossy mode in absorbing thin films. This happens due to the lossy nature of the GZO film and the matching phase between the guided mode supported by a side-polished fiber and the lossy mode supported by the GZO thin film.

\section{Experimental results}

In a single mode fiber (SMF), the cladding refractive index $n_{\text {clad }}=1.4580$ is lower than that of the core $n_{\text {core }}=1.4629$. If a part of the cladding is replaced by an external medium whose refractive index $n_{\text {ex }}$ is greater than the mode effective index $n_{\text {eff }}$, the mode becomes leaky and some of the optical power is radiated. The goal of high sensitivity can be reached by thinning the single mode fibers to produce an evanescent wave interaction of the guided light with the surrounding medium. The side-polished fibers must be polished to form a smooth surface on which a GZO thin film can be deposited. In this work, single mode fibers were polished by a homemade fiber-polishing machine. For side-polished optical fibers, the core is very close to the flat surface of the proposed structure as a side-polished fiber is thinning enough to allow evanescent wave interaction of the guided mode with the surroundings. The side-polished fiber section is more sensitive to the surrounding-medium refractive index change and is suitable for the measurement of different refractive indices in liquids, in the fabrication of side -polished fibers with different remaining thicknesses (about 72-77 $\mu \mathrm{m}$ ). The cladding was removed away until the strong evanescent field was accessible. The particle size of the polishing slurry was roughly $1 \mu \mathrm{m}$ in order to reduce the surface roughness of the fibers to avoid scattering loss. After finishing optical fiber side-polishing, the remaining thickness of the side-polished fibers was measured by using an optical microscope. The remaining thickness of the side-polished fibers was about $76.5 \mu \mathrm{m}$, and the polished length was $30 \mathrm{~mm}$. Side-polished fiber devices have a limited interaction strength because they are based on evanescent wave interaction. The proposed sensing device based on a side-polished fiber is schematically illustrated in Fig. 1. Figure 1a depicts the 3D structure of a side-polished single mode fiber. Figure $1 \mathbf{b}$ shows a conceptual diagram of a side-polishing fiber with an external medium placed on the polished flat surface of the single mode fiber. The insertion loss of such a sensor depends on the core-surface distance $d$ which is from the core center to the polished surface, and the length of fiber coated by the external medium $\left(n_{\mathrm{ex}}\right)$. We proposed a side-polished fiber with a core-surface distance of $d=14 \mu \mathrm{m}$, the radius of the core $a=4.5 \mu \mathrm{m}$ and 

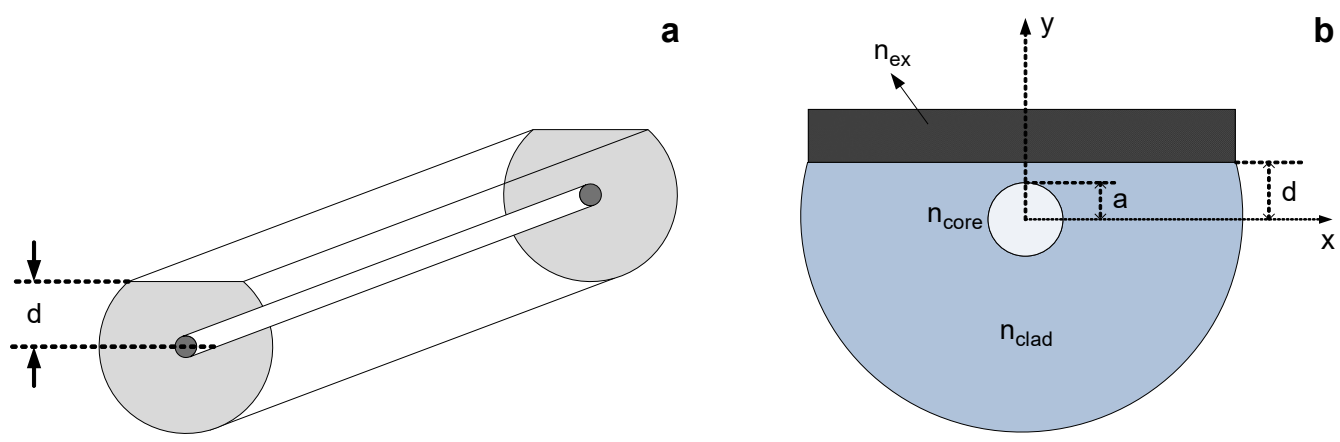

Fig. 1. The proposed side-polished fiber (a) 3D structure (b) cross-section structure diagram.

the radius of the cladding $62.5 \mu \mathrm{m}$. If $n_{\text {clad }}$ and $n_{\text {core }}$ are hold constant, the insertion loss of the side-polished fiber with $\mathrm{TE}_{0}$ mode for the LMR-based sensor is a function of $n_{\text {ex }}$.

The side-polished fibers were used as the substrate in the coating process. Before GZO thin film deposition, the partial cladding was removed and the resultant core portion was ultrasonically cleaned. After this cleaning process, a side-polished fiber was embedded and glued onto a quartz substrate. Then, GZO thin film was coated onto the side-polished surface using an electron-beam evaporation with ion assisted deposition method. The GZO film's thickness of $69 \mathrm{~nm}$ was measured using an alpha-step profilometer. Figure 2 shows the scanning electron microscopy (SEM) image of the side -polished fiber coated with GZO thin film. In the present experiment, distilled water was used to avoid any type of contamination error. Similarly, the measured solutions, having different concentrations of sodium chloride $(\mathrm{NaCl})$ solutions, i.e. $3.6 \%, 7.3 \%$, $10.9 \%, 14.6 \%, 18.2 \%$, and $21.9 \%$, were prepared for the salinity measurements. Two kinds of experiments were conducted in saline solutions. The first kind configuration was to detect the refractive indices (RI) of saline solutions from RI $=1.333$ to 1.398. The RI of saline solutions was previously determined by an Abbe refractometer with a resolution of $1 \times 10^{-4}$ for indices of 1.33 to 1.58 . The second experiment was the

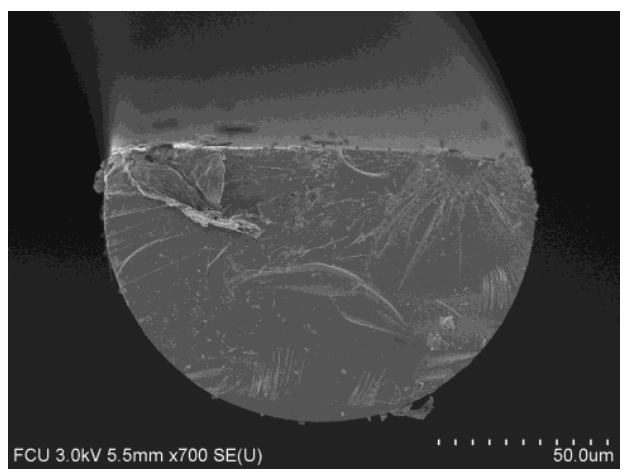

Fig. 2. SEM image of GZO thin film coated on the side-polished fiber. 


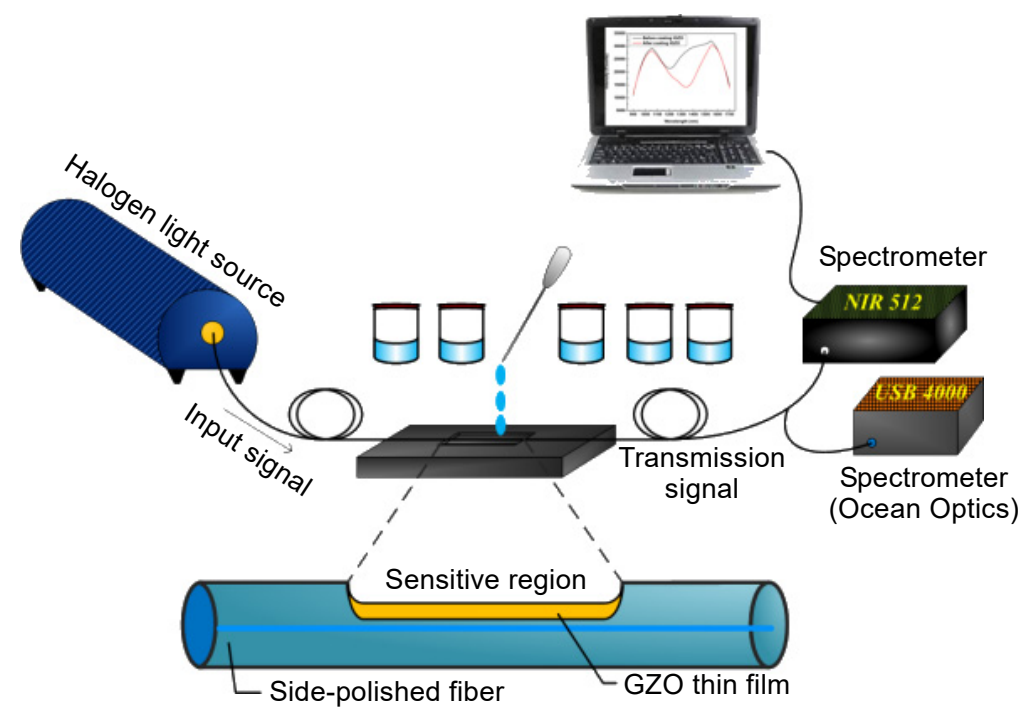

Fig. 3. Experimental setup with a white light source, spectrometers, and a side-polished fiber coated with GZO thin film.

salinity measurement by using the same LMR-based fiber sensor. The proposed fiber sensor was immersed in different concentrations of saline solutions with a range of salinities from $3.6 \%$ to $21.9 \%$. This measurement range is wider than the formerly published papers for salinity measurements. The above fiber-optic sensing device was immersed into saline solutions and the transmission spectrum data was analyzed by a computer program. The variation in the optical spectrum of the proposed LMR fiber -optic sensor with different saline solutions was measured. Figure 3 shows the experimental setup that contained a halogen light source (Ocean Optics, HL-2000) connected to a fiber-optic sensor with the other end attached to two spectrometers (Ocean Optics, USB 4000 and NIR 512). The output spectrum of two spectrometers was observed in the wavelength range of 350-1000 nm and 900-1700 nm, respectively. For the salinity measurement experiments, the saline solutions with different refractive indexes used to characterize the salinity response of the proposed fiber-optic sensor were prepared by adjusting the percent concentration of saline solutions. The sensitive section of the LMR-based sensors was immersed into saline solutions at fixed salinity indices $(3.6 \%, 7.3 \%, 10.9 \%, 14.6 \%, 18.2 \%$ and $21.9 \%)$. The salinity sensing process was measured by the spectrometers when the measured $\mathrm{NaCl}$ solutions with different salinity are filled in the measurement cell. This setup was used to detect the optical transmission output response at two different wavelength regions (visible and near infrared). Thus, the light coupled into the fiber sensor passed through the film/cladding sensitive region, located in the optical transmission pathway. We sequentially changed the saline solutions and analyzed the data measured by the USB 4000 and NIR 512 spectrometer. Repeated measurements were performed under an invariant condition to examine the performance of the proposed salinity sensor. 


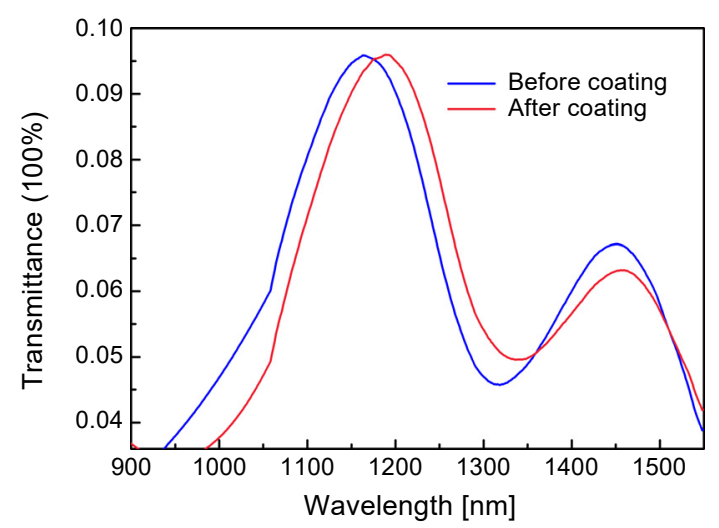

Fig. 4. Normalized transmitted spectra of the side-polished fiber before and after GZO coatings.

In this study, we can see that the wavelength shifts in the LMR effect exhibit different spectra for each concentration of saline solutions. Before the thin film deposition, the optical spectrum was taken as a reference signal. Figure 4 illustrates the optical transmission spectrum of side-polished fiber shifts from a shorter to a longer wavelength after GZO thin film coating. The results can be observed as a noticeable red -shift of the transmission spectra when the coating process is applied. The refractive index and thickness of GZO thin film can be determined by an ellipsometer. Using the LMR effect as a sensing mechanism, we can fabricate different liquid RI sensors with high sensitivity. The sensitivity of the proposed sensor was analyzed by using the wavelength interrogation method [37]. The sensitivity $S$ of the LMR-based sensor is defined as follows:

$$
S=\frac{\Delta \lambda}{\Delta n}
$$

where the resonance wavelength shift of $\Delta \lambda$ is the spectral difference between two resonance peaks (or resonance minima), and $\Delta n$ is the variation of the analyte refractive index.

The structure of a LMR-based sensing device consists of a side-polished fiber and a thin film coated on a flat surface for generating the evanescent field. As there is a resonant coupling of light to modes guided in the external coating, thus LMR could be generated. The spectrum of the transmitted light for the RI detection is recorded at the other end of the fiber. The spectral response of a side-polished fiber sensor with different RI solutions is represented in Fig. 5. We changed the refractive index of the sensing solutions in the sensing region. The proposed fiber-optic RI sensor has obviously LMR peaks (or resonance minima) when the wavelength is in the range of 1300-1600 nm. The refractive indices of the sensing solutions were measured using an Abbe refractometer. The range of the refractive indices of the sensing solutions was 1.333 to 1.398. The fiber-optic sensing region was immersed into sensing solutions, and the transmission spectrum data was saved, respectively. The variation in the optical spectrum of GZO thin 


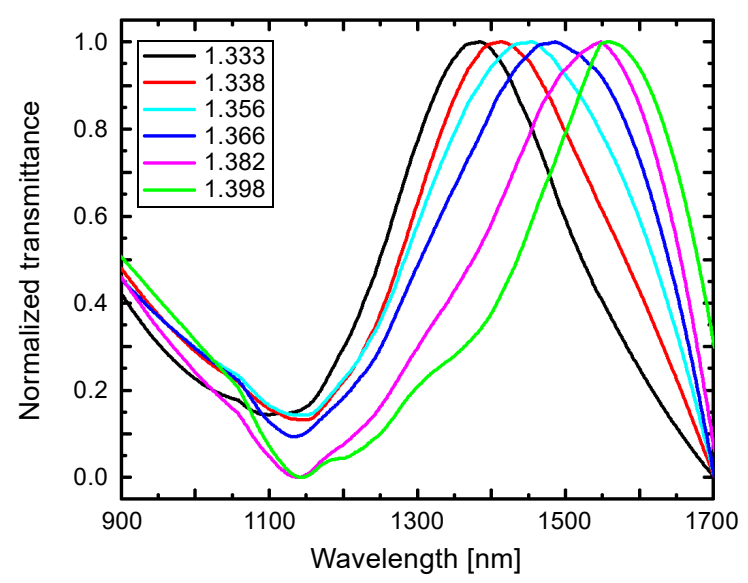

Fig. 5. Normalized transmitted spectra of LMR-based RI sensor with different saline solutions.

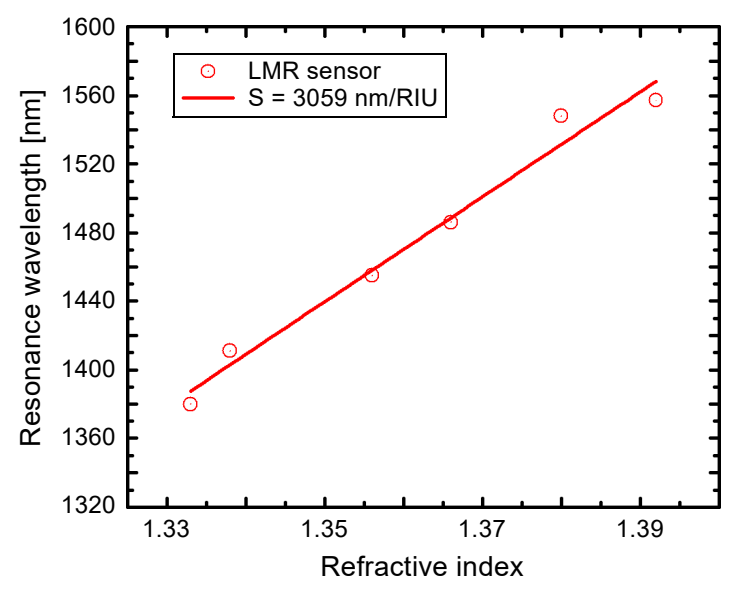

Fig. 6. The RI sensing performance of the proposed LMR-based fiber sensor.

film coated onto the side-polished fiber with different RI solutions was measured. Figure 6 shows the sensitivity of the proposed liquid RI fiber-optic sensor. The total wavelength shift of the resonance peaks was $180.5 \mathrm{~nm}$. The highest sensitivity of the proposed fiber-optic sensor was $3059 \mathrm{~nm} /$ RIU. Here RIU stands for a refractive index unit. These results show that GZO film coated on the side-polished fibers supported the generation of LMR effect. As the surrounding RI sensing solutions changed, the LMR shifted toward the longer wavelength side. The sensitivity to thickness can be expressed as a wavelength shift versus coating thickness variation. Some LMR simulation results showing several resonances are presented in Fig. 7. It can be seen that four different LMRs are generated during the thin film coating process within the thickness range of $20-400 \mathrm{~nm}$. The first LMR occurs when a film thickness is $20-100 \mathrm{~nm}$ and presents a red-shift as a function of the coating thickness. In the same manner, the 
a

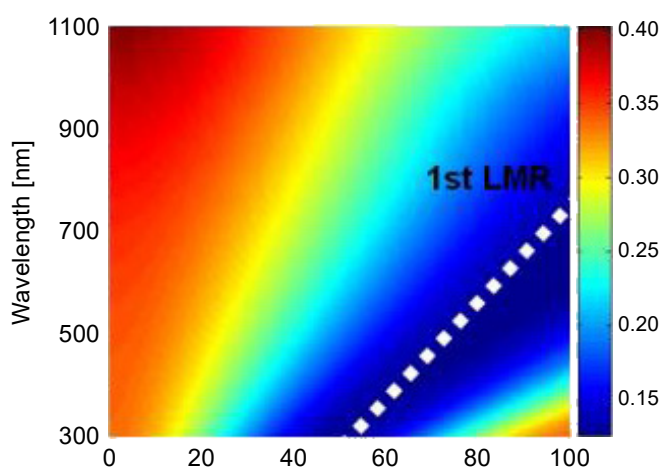

C

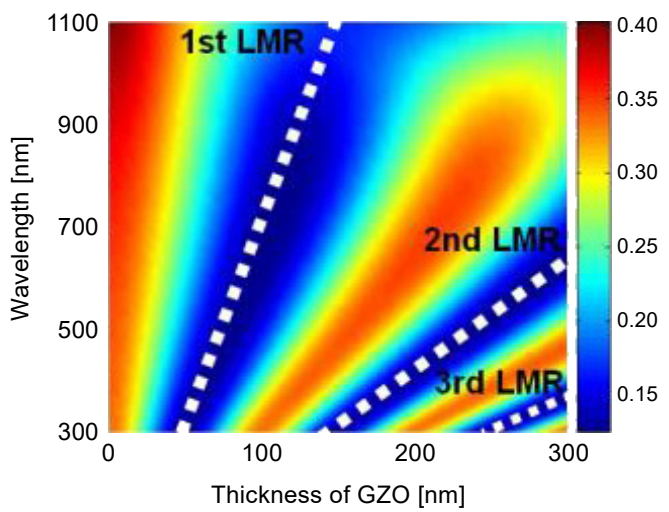

b

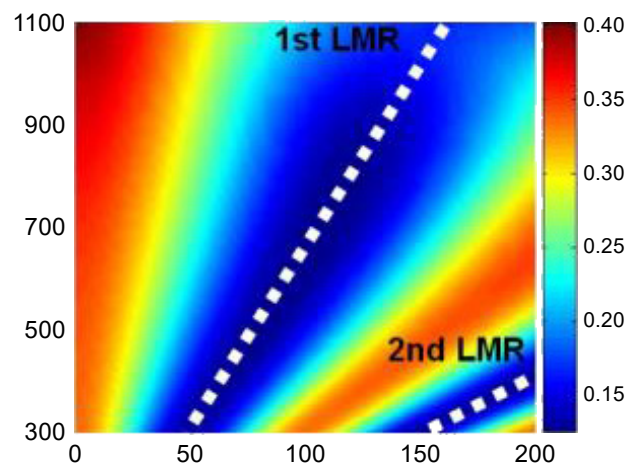

d

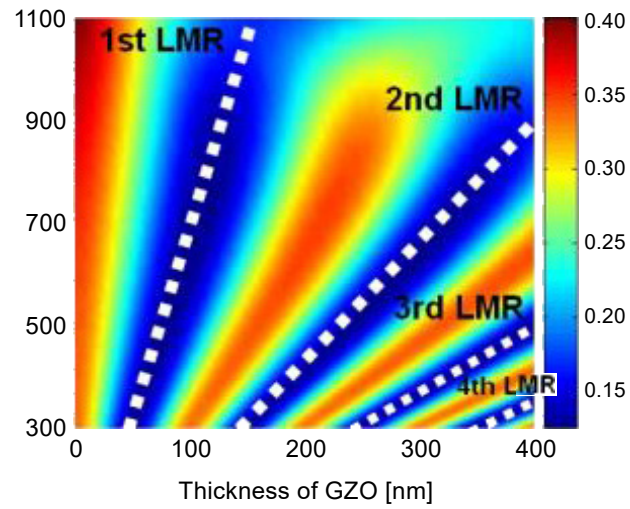

Fig. 7. LMR spectra response simulation for different GZO film thicknesses: (a) 20-100 nm, (b) 20-200 nm, (c) $20-300 \mathrm{~nm}$, (d) $20-400 \mathrm{~nm}$.

second LMR occurs at film thickness of 20-200 nm and also shifts to the longer wavelength as the coating thickness increases. The third LMR is observed around thickness of 200-300 nm. The fourth LMR occurs at film thickness of 300-400 nm. The first and the second LMRs present different spectral shifts as a function of the coating thickness. It is suggested that the first LMR is more sensitive than the second LMR for coating thickness variations. On the other hand, the experimental results reveal that the thickness of GZO thin film grew thinner, while the sensitivity of the LMR-based fiber sensor increased. Figure 8 indicates the sensitivity as a function of the GZO film's thickness. The relationship curve is fitted by a second order exponential decay function. Spectral response is obtained as a function of thickness for GZO thin film coated on side-polished fibers. In Fig. 7, the theoretical simulations represent the evolution of the LMRs generated with GZO coatings as a function of the coating thickness. As a result, the theoretical simulation agrees well with the experimental results. 


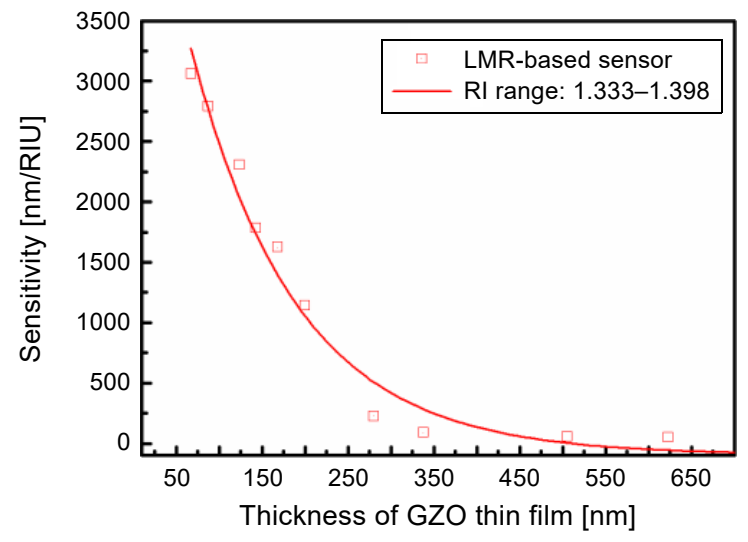

Fig. 8. The sensitivity as a function of the GZO film's thickness.

Since the refractive index of the saline solutions varies with salinity, several measuring methods of an optical fiber salinity sensor have been developed based on this sensing mechanism. Our method is based on the LMR wavelength shift due to the RI change, which is nearly proportional to the salinity. The proposed LMR-based RI sensor is able to detect the salinity variations. Figure 9 shows the transmission spectrum of the LMR -based fiber sensor as a function of the degree of salinity. This LMR-based salinity sensor indicates resonance minima (resonance dip) that have a red-shift for higher surrounding salinity indices in the salinity range of $3.6-21.9 \%$. In other words, the salinity is going up, the refractive index increases, which results in a red-shift for the transmission spectrum. It can be seen that the maximum wavelength shift in the resonance minima

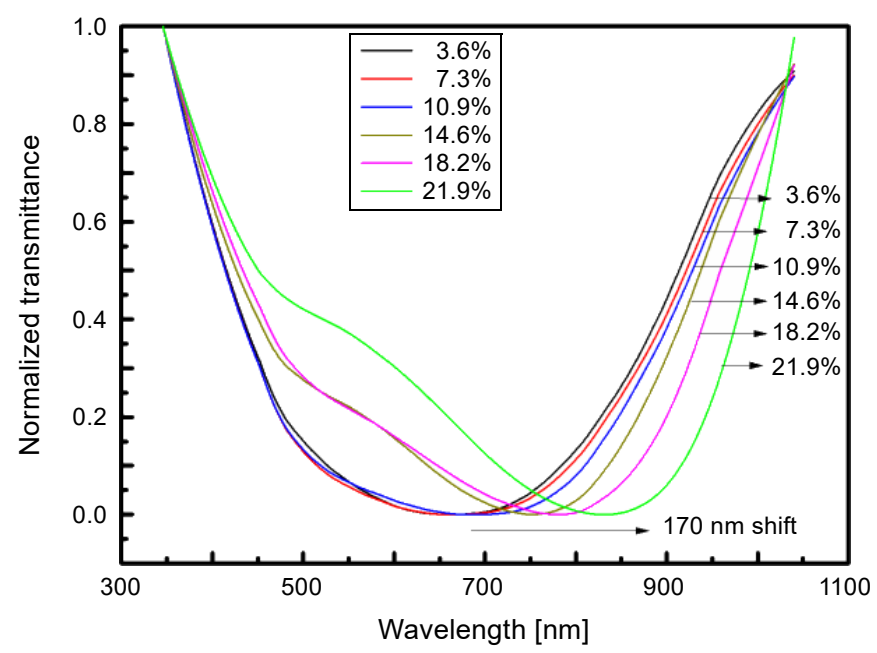

Fig. 9. The normalized transmitted spectra of the LMR-based sensor with different saline solutions. 


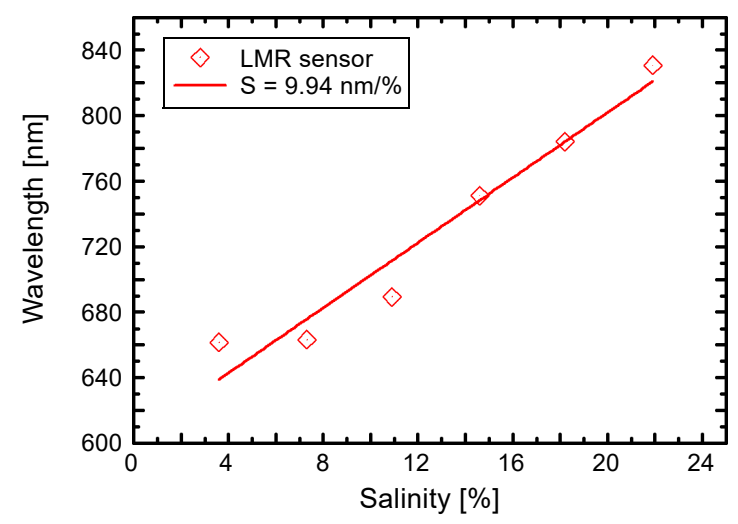

Fig. 10. The salinity sensing characteristic of the proposed LMR-based fiber sensor.

is $170 \mathrm{~nm}$. The sensitivity of the proposed LMR-based salinity sensor is $9.94 \mathrm{~nm} / \%$, as shown in Fig. 10. The experimental results reveal that our method can offer a powerful scheme for the liquid refractive index and salinity measurement. The proposed sensor can be used as a quasi-distributed salinity detector to monitor water quality in coastal areas or salinity levels in wells.

\section{Conclusions}

An experimental research about the LMR-based fiber-optic sensor has been carried out in this work. A transparent conductive oxide, like GZO thin film, has been successfully used for the LMR generation. In comparison to traditional fiber sensors, side-polished fiber has various residual thicknesses that can be tunable for better sensing performance. A side-polished fiber and GZO thin-film coating technique were used to analyze the sensing parameters. In the optical spectrum, a resonance wavelength red-shift was observed with the RI increasing. The measurement sensitivity of a LMR-based sensor can reach $3059 \mathrm{~nm} / \mathrm{RIU}$ in the RI range of $1.333-1.398$ and $9.94 \mathrm{~nm} / \%$ in the salinity range of 3.6-21.9\%. Our results demonstrate a liquid refractive index and salinity sensor with high sensitivity. Furthermore, the proposed sensor structure with easy fabrication process can be readily adapted through modification of the thin-film coatings and will help for the detection in biosensing and biomedical applications.

Acknowledgment - The authors are thankful to the Ministry of Science and Technology of Taiwan under Contract number MOST 109-2221-E-035-022, and the Ministry of Education RSC Project (20M22026) for providing financial support.

\section{References}

[1] Joe H.E., Yun H., Jo S.H., Jun M.B.G., Min B.K., A review on optical fiber sensors for environmental monitoring, International Journal of Precision Engineering and Manufacturing-Green Technology 5(1), 2018, pp. 173-191, DOI: 10.1007/s40684-018-0017-6. 
[2] Tahhan S.R., Chen R.Z., Huang S., Hajim K.I.,Chen K.P., Fabrication of fiber Bragg grating coating with $\mathrm{TiO}_{2}$ nanostructured metal oxide for refractive index sensor, Journal of Nanotechnology 2017, 2017, article 2791282, DOI: 10.1155/2017/2791282.

[3] Li Q.S., Zhang X.L., He H., Meng Q., Shi J., Wang J.N., Dong W.F., Improved detecting sensitivity of long period fiber gratings by polyelectrolyte multilayers: the effect of film structures, Optics Communications 331, 2014, pp. 39-44, DOI: 10.1016/j.optcom.2014.05.046.

[4] Hasan Md. R., Akter S., Rahman M.S., Ahmed K., Design of a surface plasmon resonance refractive index sensor with high sensitivity, Optical Engineering 56(8), 2017, article 087101, DOI: 10.1117/1.OE.56.8.087101.

[5] Usha S.P., GuPTA B.D., Performance analysis of zinc oxide-implemented lossy mode resonance-based optical fiber refractive index sensor utilizing thin film/nanostructure, Applied Optics 56(20), 2017, pp. 5716-5725, DOI: 10.1364/AO.56.005716.

[6] Gunter G., Ballard B.S., Venkataramiah A., A review of salinity problems of organisms in United States coastal areas subject to the effects of engineering works effects of engineering works, Gulf Research Reports 4(3), 1974, pp. 380-475, DOI: 10.18785/GRR.0403.05.

[7] JIN Y.Y., ChEN Z.X., WANG Q.J., Measurement laser optic-fiber technique for measuring the salinity of a solar pond, Acta Eneglae Solaris Sinica 15, 1994, pp. 198-200.

[8] Diniz F.B., De Freitas K C.S., DE Azevedo W.M., Salinity measurements with polyaniline matrix coated wire electrodes, Electrochemistry Communications 1(7), 1999, pp. 271-273, DOI: 10.1016/ S1388-2481(99)00057-0.

[9] ZHAO Y., LiAO Y., Novel optical fiber sensor for simultaneous measurement of temperature and salinity, Sensors and Actuators B: Chemical 86(1), 2002, pp. 63-67, DOI: 10.1016/S0925-4005(02)00148-X.

[10] ZhaO Y., LiaO Y., Zhang B., LaI S., Monitoring technology of salinity in water with optical fiber sensor, Journal of Lightwave Technology 21(5), 2003, pp. 1334-1338, DOI: 10.1109/JLT.2003.811318.

[11] Men L., Lu P., ChEN Q., A multiplexed fiber Bragg grating sensor for simultaneous salinity and temperature measurement, Journal of Applied Physics 103(5), 2008, article 053107, DOI: 10.1063/ 1.2890156.

[12] Qian Y., Zhao Y., Wu Q.L., Yang Y., Review of salinity measurement technology based on optical fiber sensor, Sensors and Actuators B: Chemical 260, 2018, pp. 86-105, DOI: 10.1016/j.snb.2017.12.077.

[13] Yu F.T.S., Yin S., Fiber Optic Sensors, Dekker, New York, 2002, Chaps. 2 and 4.

[14] Andreev A.T., Zafirova B.S., Karakoleva E.I., Dikovska A.O., Atanasov P.A., Highly sensitive refractometers based on a side-polished single-mode fibre coupled with a metal oxide thin-film planar waveguide, Journal of Optics A 10(3), 2008, article 035303, DOI: $\underline{10.1088 / 1464-4258 / 10 / 3 /}$ $\underline{035303 .}$.

[15] TiEn C.L., Chen H.W., Liu W.F., Jyu S.S., Lin S.W., Lin Y.S., Hydrogen sensor based on side-polished fiber Bragg gratings coated with thin palladium film, Thin Solid Films 516(16), 2008, pp. 5360-5363, DOI: $10.1016 / \mathrm{j}$. tsf.2007.07.045.

[16] Schroeder K., Ecke W., Mueller R., Willsch R., Andreev A., A fibre Bragg grating refractometer, Measurement Science and Technology 12(7), 2001, pp. 757-764, DOI: $10.1088 / 0957-0233 / 12 / 7 / 301$.

[17] Cusano A., Iadicicco A., Pilla P., Contessa L., Campopiano S., Cutolo A., Giordano M., Mode transition in high refractive index coated long period gratings, Optics Express 14(1), 2006, pp. 19 -34, DOI: 10.1364/OPEX.14.000019.

[18] Tian M., Lu P., Chen L., Lv C., Liu D., All-solid D-shaped photonic fiber sensor based on surface plasmon resonance, Optics Communications 285(6), 2012, pp. 1550-1554, DOI: 10.1016/j.optcom. 2011.11.104.

[19] Wu L., Cнu H.S., Кон W.S., Li E.P., Highly sensitive graphene biosensors based on surface plasmon resonance, Optics Express 18(14), 2010, pp. 14395-14400, DOI: 10.1364/OE.18.014395.

[20] Yang F., SAmbles J.R., Bradberry G.W., Long-range surface modes supported by thin films, Physical Review B 44(11), 1991, pp. 5855-5872, DOI: 10.1103/PhysRevB.44.5855.

[21] Kretschmann E., Raether H., Radiative decay of non radiative surface plasmons excited by light, Zeitschrift für Naturforschung A 23(12), 1968, pp. 2135-2136, DOI: 10.1515/zna-1968-1247. 
[22] Yang F., Sambles J.R., Determination of the optical permittivity and thickness of absorbing films using long range modes, Journal of Modern Optics 44(6), 1997, pp. 1155-1163, DOI: $\underline{10.1080 /}$ 09500349708230726.

[23] Wang T., Zalkovskij M., Iwaszczuk K., Lavrinenko A.V., Naik G.V., Kim J., Boltasseva A., JEPSEN P.U., Ultrabroadband terahertz conductivity of highly doped ZnO and ITO, Optical Materials Express 5(3), 2015, pp. 566-575, DOI: 10.1364/OME.5.000566.

[24] Kim J., Naik G.V., Gavrilenko A.V., Dondapati K., Gavrilenko V.I., Prokes S.M., Glembocki O.J., Shalaev V.M., Boltasseva A., Optical properties of gallium-doped zinc oxide-A low-loss plasmonic material: first-principles theory and experiment, Physical Review X 3(4), 2013, article 041037, DOI: 10.1103/PhysRevX.3.041037.

[25] Kim J.S., Jeong J.H., Park J.K., Baik Y.J., Kim I.H., Seong T.Y., Kim W.M., Optical analysis of doped $\mathrm{ZnO}$ thin films using nonparabolic conduction-band parameters, Journal of Applied Physics 111(12), 2012, article 123507, DOI: 10.1063/1.4729571.

[26] Kim Y.H., Jeong J., Lee K.S., Cheong B., Seong T.Y., Kim W.M., Effect of composition and deposition temperature on the characteristics of Ga doped $\mathrm{ZnO}$ thin films, Applied Surface Science 257(1), 2010, pp. 109-115, DOI: 10.1016/j.apsusc.2010.06.045.

[27] Del Villar I., Hernaez M., Zamarreño C.R., Sánchez P., Fernández-Valdivielso C., Arregui F.J., Matias I.R., Design rules for lossy mode resonance based sensors, Applied Optics 51(19), 2012, pp. 4298-4307, DOI: 10.1364/AO.51.004298.

[28] Gupta B.D., Verma R.K., Surface plasmon resonance-based fiber optic sensors: principle, probe designs, and some applications, Journal of Sensors 2009, 2009, article 979761, DOI: 10.1155/2009/ $\underline{979761 .}$

[29] Batchman T., McWright G., Mode coupling between dielectric and semiconductor planar waveguides, IEEE Journal of Quantum Electronics 18(4), 1982, pp. 782-788, DOI: 10.1109/JQE.1982. 1071578.

[30] Del Villar I., Zamarreno C.R., Hernaez M., Arregui F.J., Matias I.R., Lossy mode resonance generation with indium-tin-oxide-coated optical fibers for sensing applications, Journal of Lightwave Technology 28(1), 2010, pp. 111-117, DOI: 10.1109/JLT.2009.2036580.

[31] Zubiate P., Zamarreño C.R., Del Villar I., Matias I.R., Arregui F.J., High sensitive refractometers based on lossy mode resonances (LMRs) supported by ITO coated D-shaped optical fibers, Optics Express 23(6), 2015, pp. 8045-8050, DOI: 10.1364/OE.23.008045.

[32] Zhao W.M, Wang Q., Wang X.Z., Li X., Jing J.Y., Sun H.Z., Theoretical and experimental research of lossy mode resonance-based high-sensitivity optical fiber refractive index sensors, Journal of the Optical Society of America B 36(8), 2019, pp. 2069-2078, DOI: 10.1364/JOSAB.36.002069.

[33] Hernáez M., Del Villar I., Zamarreño C.R., Arregui F.J., Matias I.R., Optical fiber refractometers based on lossy mode resonances supported by $\mathrm{TiO}_{2}$ coatings, Applied Optics 49(20), 2010, pp. 3980-3985, DOI: 10.1364/AO.49.003980.

[34] Zamarreño C.R., Sanchez P., Hernáez M., Del Villar I., Fernandez-Valdivielso C., Matías I.R., ARREGUi F.J., Sensing properties of indium oxide coated optical fiber devices based on lossy mode resonances, IEEE Sensors Journal 12(1), 2012, pp. 151-155, DOI: 10.1109/JSEN.2011.2142181.

[35] Ziman M., Principles of the Theory of Solids, Cambridge University Press, 1979.

[36] Look D., Leedy K., Grzybowski G., Claflin B., Near-infrared (1 to $4 \mu \mathrm{m}$ ) control of plasmonic resonance wavelength in Ga-doped $\mathrm{ZnO}$, Optical Engineering 56(5), 2017, article 057109, DOI: 10.1117/1.OE.56.5.057109.

[37] Chen H., Zhang S., Fu H., Zhou B., Chen N., Sensing interrogation technique for fiber-optic interferometer type of sensors based on a single-passband RF filter, Optics Express 24(3), 2016, pp. 2765 -2773, DOI: 10.1364/OE.24.002765. 